Planetary Systems in the Universe - Observation, Formation and Evolution Proceedings IAU Symposium No. 202, (C2004 IAU

Alan Penny, Pawel Artymowicz, Anne-Marie Lagrange, $\mathcal{G}$ Sara Russell, eds.

\title{
Gas temperatures in Vega-type stars
}

\author{
Iain M. Coulson \\ Joint Astronomy Centre, Hilo, Hawaii, 96720, U.S.A.
}

Dolores M. Walther

Gemini $8 m$ Telescope Project, Hilo, Hawaii, 96720, U.S.A.

William R.F. Dent

Royal Observatory Edinburgh, Edinburgh EH9 3HJ, U.K.

\begin{abstract}
Further observations of the submillimetre CO lines in 3 Vega-type stars are reported and interpreted for the gas temperatures they imply. Very low gas temperatures $(\sim 15 \mathrm{~K})$ are deduced for all three stars, consolidating the suggestion that, if the gas and dust are remnants of the molecular cloud from which the star system formed, then much of the $\mathrm{CO}$ in these systems may be locked up in ices on the surfaces of dust grains.
\end{abstract}

\section{Introduction}

Vega-type stars are main-sequence stars showing unexpected infrared emission (Aumann et al 1984) presumably coming from a dusty disk. Submillimetre imaging of the nearby, archetype systems such as Vega and Fomalhaut (Holland et al 1998) and Eps Eri (Greaves et al 1999) reveals no standard geometries for the cold dust, and so we should be hesitant yet in generalizing about these systems. There is a sub-class of dustier systems, typified by SAO206462 (Coulson \& Walther 1995), but they are generally too distant to resolve with the relatively large beam of the JCMT. The 3 stars in this work belong to this subclass. Searches for accompanying CO gas have found none in the archetypes (Yamashita et al 1993, Dent et al 1995). A previous analysis of the $\mathrm{J}=2-1$ and $\mathrm{J}=3-2$ lines of $\mathrm{CO}$ in SAO206462 (HD135344, Coulson et al 1999) yielded a gas temperature in conflict with the dust temperature available at that time. We present here $\mathrm{J}=2-1$ and $\mathrm{J}=3-2$ detections in 2 other dusty Vegas, as well as a $\mathrm{J}=4-3$ detection in SAO206462, and derive their $\mathrm{CO}$ gas temperatures for comparison with newly derived dust temperatures.

\section{Data}

The CO spectroscopic data reported here have been collected over several years at the James Clerk Maxwell submillimetre telescope. The JCMT has a suite of heterodyne receivers using SIS mixer junctions designed to work in the transparent bands of the submillimetre spectrum centred at $230 \mathrm{GHz}$ (A-band), 
$345 \mathrm{GHz}(\mathrm{B})$ and $470 \mathrm{GHz}(\mathrm{C})$. The $\mathrm{J}=2-1, \mathrm{~J}=3-2$ and $\mathrm{J}=4-3$ rotational lines of $\mathrm{CO}$ lie conveniently in the centres of these bands.

Table 1. JCMT parameters

$\begin{array}{cccc}\text { CO line } & \text { Freq.(GHz) } & \text { HPBW (arcseconds) } & \eta_{\text {beam }} \\ & & & \\ \mathrm{J}=2-1 & 230 & 21 & 0.63 \\ \mathrm{~J}=3-2 & 345 & 14 & 0.56 \\ \mathrm{~J}=4-3 & 461 & 11 & 0.5\end{array}$

Integrations for these observations are typically an hour or more per transition. Data are collected using a beamswitching technique, chopping at $8 \mathrm{~Hz}$ against the sky 60 or 90 arcseconds away in azimuth. The IF signal from each receiver is sent to the DAS autocorrelation spectrometer which can be configured to give resolutions as good as $0.075 \mathrm{MHz}(0.1 \mathrm{~km} / \mathrm{s})$. The data are displayed uniformly here at a resolution of $1 \mathrm{~km} / \mathrm{s}$. Calibration is by reference to standard spectra of astronomical sources. Line fitting has been done using the SPECX package at JCMT. Observations were also taken off-source of SAO112630 (4 points, below, left) and SAO206462 (one point) that confirm the stars, rather than any other objects in the line-of-sight, as the source of these emissions.
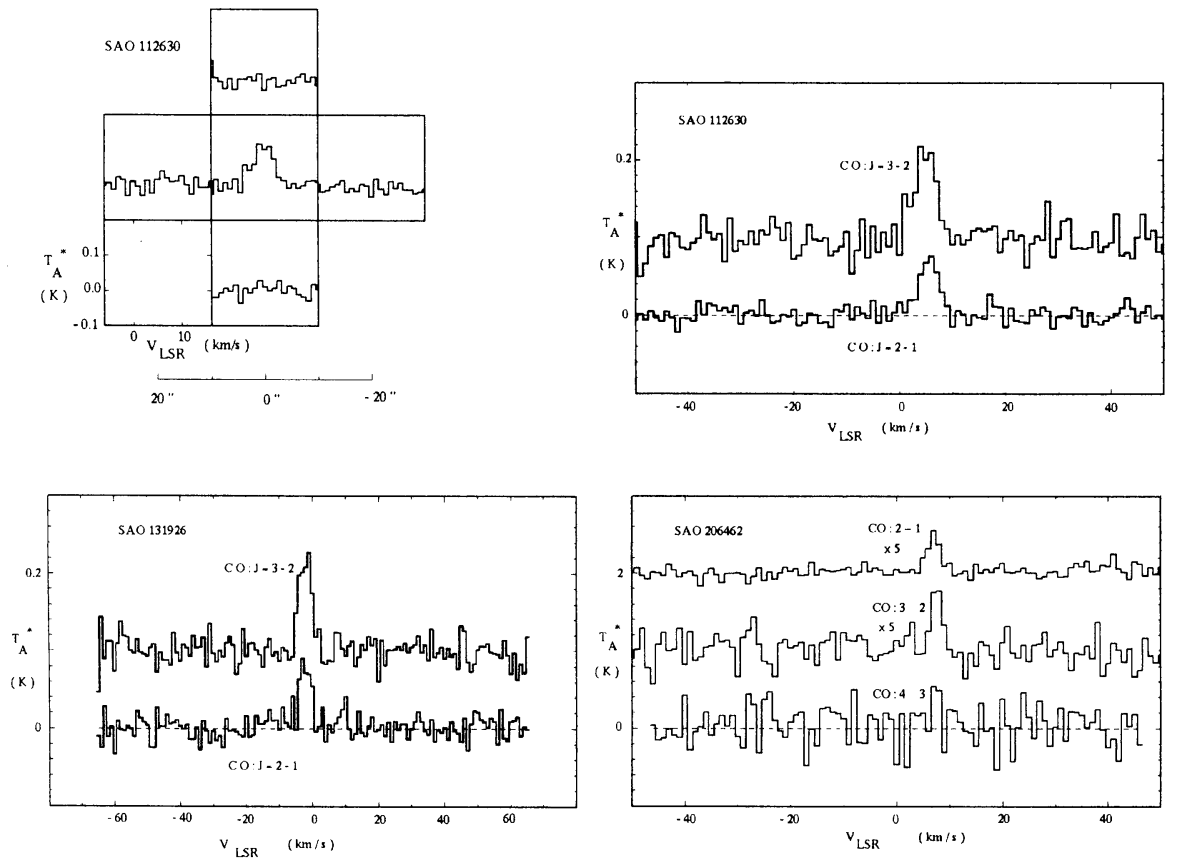
Table 2. CO line intensities and gas temperatures for 3 Vega-type stars

\begin{tabular}{|c|c|c|c|c|c|c|c|}
\hline $\begin{array}{l}\mathrm{SAO} \\
(\mathrm{HD})\end{array}$ & $\begin{array}{l}\mathrm{CO} \\
\text { line }\end{array}$ & $\begin{array}{c}\mathrm{T}_{A \text { peak }}^{*} \\
\mathrm{~K}\end{array}$ & $\begin{array}{c}\text { FWHM } \\
\mathrm{km} / \mathrm{s}\end{array}$ & $\begin{array}{c}\mathrm{Vel}_{L S R} \\
\mathrm{~km} / \mathrm{s}\end{array}$ & $\begin{array}{l}\mathrm{T}_{A}{ }^{*} \mathrm{dv} \\
\mathrm{Kkm} / \mathrm{s}\end{array}$ & $\begin{array}{c}\mathrm{T}_{C O} \\
\mathrm{~K}\end{array}$ & $\begin{array}{c}\mathrm{T}_{\text {dust }} \\
\mathrm{K}\end{array}$ \\
\hline $\begin{array}{l}112630 \\
(34700)\end{array}$ & $\begin{array}{l}\mathrm{J}=2-1 \\
\mathrm{~J}=3-2\end{array}$ & $\begin{array}{l}0.08 \\
0.12\end{array}$ & $\begin{array}{l}4.1 \\
4.8\end{array}$ & $\begin{array}{l}5.6 \\
4.9\end{array}$ & $\begin{array}{l}0.33 \pm 0.04 \\
0.60 \pm 0.09\end{array}$ & 18 & 75 \\
\hline $\begin{array}{l}131926 \\
(34282)\end{array}$ & $\begin{array}{l}\mathrm{J}=2-1 \\
\mathrm{~J}=3-2\end{array}$ & $\begin{array}{l}0.09 \\
0.13\end{array}$ & $\begin{array}{l}4.3 \\
4.5\end{array}$ & $\begin{array}{l}-2.3 \\
-2.1\end{array}$ & $\begin{array}{l}0.40 \pm 0.06 \\
0.61 \pm 0.07\end{array}$ & 15 & 27 \\
\hline $\begin{array}{c}206462 \\
(135344)\end{array}$ & $\begin{array}{l}\mathrm{J}=2-1 \\
\mathrm{~J}=3-2 \\
\mathrm{~J}=4-3\end{array}$ & $\begin{array}{c}0.11 \\
0.17 \\
0.7\end{array}$ & $\begin{array}{l}2.5 \\
2.4 \\
1.2\end{array}$ & $\begin{array}{l}7.1 \\
7.5 \\
7.5\end{array}$ & $\begin{array}{c}0.35 \pm 0.05 \\
0.42 \pm 0.09 \\
0.8 \pm 0.2\end{array}$ & 13 & 28 \\
\hline
\end{tabular}

\section{Line ratios and temperature determinations}

The observed line intensities, $\mathrm{T}_{A}^{*} \mathrm{dv}$, must be corrected for the dilution factor, $\mathrm{f}$, and the beam efficiency, $\eta_{\text {beam }}$ to yield the brightness temperatures, $\mathrm{T}_{R}$ : $\frac{1}{f}=1-2^{-\left(\frac{D}{H P B W}\right)^{2}}$, where $\mathrm{D}$ is the diameter of the source (typically $<1^{\prime \prime}$ ), and $T_{R}=T_{A}^{*} \frac{f}{\eta_{\text {beam }}}$. So the line ratio $\frac{T_{R}(3-2)}{T_{R}(2-1)}=\frac{T_{A}^{*}(3-2)}{T_{A}^{*}(2-1)} \frac{f_{3-2}}{f_{2-1}} \frac{\eta_{2-1}}{\eta_{3-2}}$, and when D is small $\frac{f_{3-2}}{f_{2-1}}=\left(\frac{H P B W_{2-1}}{H P B W_{3-2}}\right)^{2}$, so at JCMT $\frac{T_{R}(3-2)}{T_{R}(2-1)}=0.50 \frac{T_{A}^{*}(3-2)}{T_{A}^{*}(2-1)} \quad$. If the CO gas is optically thin and in LTE the intensity of a line from the $\mathrm{J}^{\text {th }}$ level is $\propto J^{2} e^{-2.766 \frac{J(J+1)}{T_{C O}}}$, and so $\frac{T_{R}(3-2)}{T_{R}(2-1)}=2.25 e^{\frac{-16.59}{T_{C O}}}$, allowing us to determine the excitation temperature, $\mathrm{T}_{C O}$, from the observed ratios. The CO:J=4-3 data proved insufficiently accurate to confirm the gas temperature for SAO206462.

\section{Discussion}

For 2 of these 3 stars the temperature of the $\mathrm{CO}$ gas is comparable with that of the cold dust, validating the assumptions of LTE and optical thinness. The $\mathrm{CO}$ gas temperatures are similar to the sublimation temperature of $\mathrm{CO}(\lesssim 20 \mathrm{~K})$, supporting the possibility that, if the gas and dust are remnants of the molecular cloud from which these systems formed, then any other $\mathrm{CO}$ in the system must be locked up as ice on the surface of the dust grains.

\section{References}

Aumann et al 1984, ApJ 278, L23

Coulson \& Walther, 1995, MNRAS 274, 977

Coulson, Walther \& Dent 1999, Astrochemistry . . IAU Symp 197, poster

Dent et al 1995, MNRAS 277, L25

Greaves et al 1999, ApJ 506, L133

Holland et al 1998, Nature 392, 788

Yamashita et al 1993, ApJ 402, L65 